\title{
O desempenho de alunos dos Cursos Pré-vestibulares Comunitários no ENEM 2006: análise de um possível impacto da capacitação de professores
}

\section{Resumo}

$\bigcirc$ artigo discute os resultados obtidos pelos alunos dos Cursos Pré-Vestibulares Comunitários do Rio de Janeiro, no ENEM 2006. Os 350 professores desses alunos, todos voluntários, participaram de atividades de Capacitação voltadas para o desenvolvimento das habilidades do ENEM. Os alunos desses professores, além de material didático, realizaram um Simulado do ENEM, em junho de 2006. Pesquisando os resultados desses alunos, no ENEM, constatou-se que eles obtiveram médias maiores do que as obtidas pelos alunos brasileiros e do Rio de Janeiro. Os alunos dos Pré-Vestibulares Comunitários, têm renda familiar menor, são mais velhos e, em maior percentual, se declararam pretos e pardos, quando comparados com os alunos brasileiros e do Rio de Janeiro. Essas variáveis, em geral, estão associadas a piores desempenhos. Os autores discutem o possível impacto da capacitação dos professores nos resultados positivos obtidos pelos alunos no ENEM 2006.
Palavras-chave: Pré-Vestibulares Comunitários. Resultados no ENEM. Impacto de capacitação de professores.

\section{Abstract}

The performance of students from Community Center Courses for admission in higher education in rio de janeiro via ENEM 2006: analysis of a possible impact of teachers'

\section{empowerment}

This article presents and analyses the students results from Community Courses for Admission in Higher Education obtained in the Brazilian National Exam of Secondary Education (ENEM) 2006. Volunteer 
teachers, in a total of 350, were trained to be able to develop ENEM abilities in their classes. Their students received classes and instructional materials related to those abilities and also took a simulated exam with 42 questions from former ENEM. Results showed that these students reached greater means than those presented by the Brazilian and Rio de Janeiro peers in the 2006 Exam. Socioeconomic variables like family income, age and ethnic groups were controlled and it was verified that students' means were higher when compared to the Brazilian means in the same strata. In general, those mentioned variables are associated to students' lower results. The authors consider students good performance as a possible impact of their teachers' empowerment through the specific training and students motivation as well.

Keywords: Community Courses for Admission in Higher Education. ENEM results. The Empowerment of teachers

\section{Resumen}

\section{El desarrollo de los} alumnos de los Cursos Preparatorios

\section{Comunitarios para} ingreso en la universidad en el examen nacional ENEM 2006: análisis de un posible impacto en la capacitación de los maestros

El artículo discute los resultados obtenidos por los alumnos de los Cursos Preparatorios Comunitarios del Rio de Janeiro para el ingreso en la Universidad a través del examen nacional ENEM 2006. Los 350 maestros de eses alumnos, todos voluntarios, participaran de actividades de capacitación para el desarrollo de habilidades presentadas en el examen ENEM. Los alumnos de eses maestros recibieron material didáctico y hicieron una prueba simulada del ENEM en junio de 2006. Los resultados de eses alumnos, en la prueba ENEM, mostró que ellos tuvieron medias superiores a de los estudiantes brasileños y del Rio de Janeiro. Los alumnos de eses cursos comunitarios tienen renta familiar más baja, son mayores y, en porcentual más elevado, se declaran negros y pardos, cuando comparados con los estudiantes brasileños y del Rio de Janeiro. Esas variables, en general, están asociadas a los peores desempeños. Los autores discuten el posible impacto de la capacitación de los maestros en los resultados positivos obtenidos por los alumnos en el ENEM 2006.

Palabras clave: Cursos Preparatorios Comunitarios para Ingreso en la Universidad. Resultados del examen ENEM. Impacto en la capacitación de maestros.

\section{O universo dos 97}

\section{cursos pré-vestibulares} comunitários do Grande Rio

Os cursos pré-vestibulares comunitários - CPVCs -, são movimentos sociopolíticos de empoderamento, diversidade cultural, cidadania ativa e instituições para-escolares, lutando por ações inclusivas no Ensino Superior. Esses cursos cresceram exponencialmente em todo o Brasil como movimentos sociais da comunidade, grupos de excluídos e pobres voltados para resgatar a 
cidadania educacional. Apesar de suas dimensões étnicas, culturais e políticas, no plano pedagógico os CPVCs têm condições objetivas bastante precárias, pois contam com recursos humanos, físicos, financeiros e técnicos muito limitados. A maioria deles opera em espaços físicos e horários cedidos em escolas públicas, privadas, sedes de associações de moradores, de igrejas e de ong's. Seu corpo docente é composto majoritariamente por voluntários e leigos, muitos ainda em formação de nível superior.

A partir de 1994, ingressam, nos cursos de Graduação do Grande Rio, os primeiros alunos oriundos dos CPVCs e, apesar do apoio dado, como no caso da Pontifícia Universidade Católica do Rio de Janeiro, em termos de cotas de bolsas integrais, auxílios-transporte, refeição e material escolar, aliados a algumas iniciativas acadêmicas de apoio psicopedagógico e de reforço escolar, ainda permanecem desafiantes à efetiva inclusão desses prévestibulandos na vida acadêmica.

Os desafios são maiores quando se considera que, desde 2004, o MEC vincula a políitica do Programa de Universidade para Todos PROUNI aos resultados do Exame Nacional de Ensino Médio - ENEM. A elevação do nível de aprendizagem dos alunos dos CPVCs torna-se indispensável para aumentar as chances objetivas de acesso via PROUNI e também para assegurar um melhor rendimento no Ensino Superior. Nesse contexto, um apoio efetivo aos CPVCs precisa considerar a formação proporcionada por esses cursos aos seus alunos para ampliar suas propostas de ensino-aprendizagem, currículo e, sobretudo, a formação docente.

A relevância sociopolítica da formação dos docentes dos CPVCs é ressaltada pelos retratos estatísticos gerados a partir de dois surveys de- senvolvidos em 2004-2005 (CARVALHO, 2006). $\bigcirc$ primeiro deles, junto ao corpo docente, aponta as condições pedagógicas desafiadoras enfrentadas por eles, além de desenhar os seus perfis. Entre os docentes e coordenadores pedagógicos, 53\% são leigos e voluntários, 17,2\% são vestibulandos e 3,2\%, estudantes secundaristas. Na distribuição desses docentes por área de conhecimento, constata-se a predominância de diplomados em Ciências Humanas $(60,4 \%)$, Ciências Exatas e Tecnológicas $(24,5 \%)$ e Ciências Sociais, (12,3\%).

segundo surveys foi realizado junto a 2783 pré-vestibulandos comunitários que participaram de uma prova simulada do ENEM em 2005. Seus resultados mostraram que $25 \%$ se encontram na faixa etária própria de pré-vestibular, enquanto 39\% tinham idade superior à prevista para conclusão do ensino universitário. Em termos de capital cultural familiar, $51 \%$ dos pais tinham apenas escolaridade fundamental e 38\% das mães haviam concluído somente o antigo primário. Quase 50\% declararam uma renda familiar inferior a 2 salários mínimos. A defasagem entre a conclusão do Ensino Médio e o curso Pré-vestibular era de cerca de 10 anos para $20 \%$ dos pré-vestibulandos comunitários, enquanto $29 \%$ deles estavam afastados dos estudos por um período de 3 a 5 anos.

\section{A estratégia delineada em 2005-2006 pela Parceria PUC-Rio/ Fundação Cesgranrio/CPVCs}

Em um primeiro momento, entre abril e julho de 2005, as precárias condições pedagógicas predominantes nos CPVC - 53\% de docentes leigos voluntários; excessiva focalização propedêutica na abordagem 
curricular, nos processos ensino-aprendizagem e na sistemática de avaliação calcada em "simuladões"; o quase inexistente processo de formação docente em serviço - foram atendidas através de um curso introdutório sobre a Matriz de Competências do ENEM. Buscou-se assim sensibilizar as Coordenações Pedagógicas dos CPVCs parceiros sobre as contribuições potenciais do ENEM, com destaque para:

> aplicação de um teste simulado do ENEM, composto por 42 questões (2 por habilidade) retiradas do Banco de Itens do ENEM para fins de efeitodemonstração. Os quase 3.000 prévestibulandos comunitários teriam assim oportunidade de exercitar previamente as competências e habilidades requeridas pelo modelo pedagógico, quais sejam interdisciplinaridade; recursos a múltiplas linguagens implicadas; questões contextualizadas, demandando diferentes mobilizações de conhecimentos e saberes; capacidade para identificar, escolher e utilizar os meios disponíveis e ainda o estabelecimento das diversas relações necessárias à solução de situações problemáticas;

efetivação do processo de empoderamento vivenciado pelos 2.800 prévestibulandos comunitários ao receberem, em seus endereços domiciliares, boletins individuais, reportando seus acertos e erros em cada item de uma prova simulada, podendo posteriormente, em classe, socializar com seus colegas e professores dos CPVCs, as condições de erros e acertos observados. Segundo relatórios dos CPVCs, esta segunda dimensão de um processo pedagógico associado ao ENEM evidenciou um impacto muito positivo ao senso de empoderamento político-educacional por esses alunos comunitários terem vivenciado a prestação de um serviço pedagógico sui-generis em suas trajetórias escolares.

No biênio 2005-2006, esta co-parceria permitiu gerar um banco de dados, contendo os itens do teste simulado sobre o ENEM e ensejando quatro produtos de pesquisas associadas:

$>$ geração de um relatório técnico sobre cada item do teste do simulado, contemplando a análise dos padrões de acerto e erro, ressaltando as competências e habilidades envolvidas em cada item, as estatísticas clássicas do teste (índice de dificuldade, índice de discriminação, coeficientes bisseriais).

$>$ a publicação de um artigo na revista Cadernos de Pesquisa da Fundação Carlos Chagas, intitulado "Os Cursos Pré-Vestibulares Comunitários e seus condicionantes pedagógicos" (CARVALHO, 2006).

$>$ um banco de dados, contemplado em uma tese de doutoramento, explorando diversas hipóteses associadas às constatações empíricas dos resultados de itens de Ciências - em especial relativos à Biologia e Meio-Ambiente - evidenciando que os pré-vestibulandos comunitários demonstraram, em todos os itens, padrões de rendimentos superiores à média nacional do ENEM, apesar das condições precárias do processo ensino-aprendizagem existentes nos CPVCs.

um relatório técnico individualizado para cada CPVC, analisando os padrões de erros e acertos dos seus 
alunos, ressaltando diversas estatísticas descritivas tanto dos itens quanto do desempenho global dos alunos de cada CPVC.

Em 2006, uma opção dos parceiros institucionais foi a de desenvolver uma formação docente em serviço, com um curso de aperfeiçoamento de 114 horas, ao longo de seis jornadas (48 horas de aulas presenciais) e de 64 horas-aula sob a forma de oficinas e de exercícios práticos junto às próprias classes comunitárias nos CPVCs, atendendo a 350 docentes voluntários de 97 CPVCs. Foi desenvolvida uma programação teórico-prática sobre as habilidades e competências do ENEM, nas seguintes áreas temáticas: i) Língua Portuguesa e Redação; ii) Matemática; iii) História; iv) Geografia; v) Física; vi) Química; vii) Biologia; viii) Coordenação Pedagógica de CPVCs.

A outra opção de apoio aos CPVCs foi a realização de uma prova simulada do ENEM no final de semana de 8 e 9 de juIho, composta por 42 itens de teste (2 questões por habilidade) e uma redação. A prova alcançou 2.400 alunos comunitários, com o posterior retorno aos quadros docentes e coordenadores dos CPVCs de um relatório técnico, consubstanciando a análise de cada item da prova simulada, seus padrões de acertos e erros, estatísticas descritivas sobre cada item. Em complementação cada CPVC teve docentes habilitados a desenvolverem a avaliação da prova de redação de seus alunos.

A disseminação dos resultados foi feita junto aos docentes em um seminário de análise de desempenho dos alunos. E foi elaborado um Relatório Técnico, no qual cada item da prova simulada foi analisado conforme os pressupostos de competências e habilidades da Matriz do ENEM, explicitando, através de estatísticas descritivas e de índices discriminantes, os diversos perfis de acertos e erros dos 2.400 pré-vestibulandos. Complementarmente, a Fundação Cesgranrio gerou para cada um desses prévestibulandos comunitários um boletim com os seus resultados individuais comparativamente às médias do ENEM nacional.

Em relação à avaliação dos resultados, por ocasião da prova simulada do ENEM, em seminário realizado em 9 de julho de 2006, o processo de formação em serviço dos quadros docentes dos CPVCs consubstanciou um expressivo ganho em duas dimensões:

$>$ quantitativamente, 65 docentes de Língua Portuguesa dos CPVCs foram devidamente habilitados pela equipe de consultores da Fundação Cesgranrio no desenvolvimento da habilidade de produção de texto e correção de redação utilizando os Critérios de Correção de Redações do ENEM;

qualitativamente, o processo de oficinas com os alunos e aplicação de testes formativos desenvolvido em termos das competências e habilidades, apresentou impactos extremamente positivos no desempenho dos alunos, evidenciado pelos maiores índices de dificuldade (percentuais de acertos) nos itens em relação ao ENEM, conforme demonstrado no quadro a seguir. Das 42 questões aplicadas, quase $50 \%$ delas (20 questões) apresentaram uma diferença positiva que variou entre 5 a 18 pontos percentuais. 


\begin{tabular}{|c|c|c|c|c|c|}
\hline $\begin{array}{l}\text { No da questão } \\
\text { Simulado }\end{array}$ & $\begin{array}{c}\text { No da questão } \\
\text { ENEM/Ano }\end{array}$ & $\begin{array}{c}N^{\circ} \text { da } \\
\text { Habilidade }\end{array}$ & $\begin{array}{l}\text { Índice de } \\
\text { dificuldade } \\
\text { Simulado }\end{array}$ & $\begin{array}{l}\text { Índice de } \\
\text { dificuldade } \\
\text { ENEM }\end{array}$ & $\begin{array}{l}\text { Diferença } \\
\text { em ponto }\end{array}$ \\
\hline 3 & $21-2002$ & 20 & .60 & .53 & +7 \\
\hline 4 & $1-2005$ & 5 & .64 & .52 & +12 \\
\hline 5 & $2-2005$ & 6 & .73 & .61 & +12 \\
\hline 6 & $10-2005$ & 19 & .46 & .40 & +6 \\
\hline 10 & $27-2001$ & 6 & .60 & .49 & +11 \\
\hline 14 & $39-2002$ & 21 & .71 & .58 & +13 \\
\hline 15 & $44-2002$ & 12 & .66 & .54 & +12 \\
\hline 16 & $42-2001$ & 5 & .64 & .54 & +10 \\
\hline 17 & $12-2005$ & 2 & .67 & .62 & +5 \\
\hline 18 & $53-2002$ & 20 & .43 & .36 & +7 \\
\hline 19 & $62-2002$ & 21 & .75 & .57 & +18 \\
\hline 20 & $63-2002$ & 18 & .44 & .33 & +11 \\
\hline 23 & $23-2003$ & 12 & .39 & .31 & +8 \\
\hline 26 & $22-2005$ & 9 & .47 & .42 & +5 \\
\hline 29 & $28-2005$ & 13 & .71 & .60 & +11 \\
\hline 32 & $35-2005$ & 10 & .44 & .38 & +6 \\
\hline 35 & $44-2005$ & 4 & .83 & .71 & +12 \\
\hline 39 & $49-2005$ & 2 & .48 & .39 & +9 \\
\hline 40 & $15-2001$ & 11 & .50 & .45 & +5 \\
\hline 41 & $20-2001$ & 4 & .56 & .48 & +8 \\
\hline
\end{tabular}

Quadro 1 - Diferença entre resultados das questões no Simulado dos CPVCs e no ENEM-Brasil.

A análise das questões revela que os ganhos são mais expressivos nas habilidades que envolvem interpretação de textos, análise de gráficos, leitura e compreensão de textos e tabelas. No entanto, nas questões mais especificamente ligadas às habilidades 10 (utilizar e interpretar diferentes escalas de tempo para situar e descrever transformações na atmosfera, biosfera, hidrosfera e litosfera, origem e evolução da vida, variações populacionais e modificações no espaço geográfico), 17 (na obtenção e produção de materiais e de insumos energéticos, identificar etapas, calcular rendimentos, taxas e índices, e analisar implicações sociais, econômicas e ambientais), 7 (identificar e caracterizar a consenvação e as transformações de energia em diferentes processos de sua geração e uso social, e comparar diferentes recursos e opções energéticas); e 14 (diante da diversidade de formas geométricas planas e espaciais, presentes na natureza ou imaginadas, caracterizá-las por meio de propriedades, relacionar seus elementos, calcular comprimentos, áreas ou volumes e utilizar o conhecimento geométrico para leitura, compreensão e ação sobre a realidade) os alunos dos CPVCs apresentaram um desempenho abaixo da média obtida pelos candidatos do ENEM no ano em que as questões foram aplicadas. 
Esses resultados estimularam a equipe do projeto a aprofundar a formação docente e propor para o primeiro semestre de 2007 a formação de professores com ênfase nas habilidades onde os alunos (e provavelmente eles próprios) têm maior dificuldade de aprendizagem, seja pela complexidade da natureza da habilidade ou pela dificuldade de romper a cadeia de pré-requisitos não adquiridos.

\section{O Desempenho dos alunos dos CPVCs no ENEM 2006}

Com o objetivo de avaliar os possíveis impactos da capacitação docente no desempenho dos alunos dos CPVCs no ENEM 2006 foram analisados os resultados dos alunos dos CPVCs que se inscreveram para o simulado em junho. É importante lembrar que o quantitativo de alunos dos CPVCs que fizeram o ENEM é maior do que os estudados já que a obtenção de dados de inscrição no exame - que possibilitaria acessar o banco de dados do INEP, em 2007 de alunos que já não freqüentavam mais os CPVCs - revelou-se uma tarefa bastante complexa. Assim, a presente pesquisa analisará o desempenho e variáveis socioeconômicas de interesse, dos alunos previamente cadastrados na prova simulada que fizeram o teste do ENEM 2006.

No cadastro de inscrição no simulado de junho, foram identificados 2577 indivíduos que também se inscreveram no ENEM. Desses, 1870 fizeram ambas as provas, 476 fizeram somente o ENEM, 132 somente o simulado e 99 não fizeram nenhuma das duas provas. Os resultados apresentados aqui se referem aos 2346 candidatos que fizeram o ENEM (ver tabela 1). Pode-se ver também que a média na prova objetiva dos que fizeram o simulado e o ENEM é maior que a dos que só fizeram o ENEM.

Tabela 1 - Cruzamento dos inscritos pela presença ou não no simulado e ENEM e média da prova objetiva no ENEM

\begin{tabular}{|l|r|r|r|r|}
\hline & \multicolumn{4}{|c|}{ Enem } \\
\hline Pré & $\mathrm{F}$ & $\mathrm{P}$ & Total & $\begin{array}{r}\text { Média } \\
\text { Enem }\end{array}$ \\
\hline Faltoso & 99 & 476 & 575 & 38,5 \\
\hline Presente & 132 & 1870 & 2002 & 40,2 \\
\hline Total & 231 & 2346 & 2577 & 39,9 \\
\hline
\end{tabular}

O ENEM é um exame nacional voluntário e gratuito para alunos da rede pública de ensino (KLEIN; FONTANIVE; ELLIOT, 2007). Em 2006 já estava na sua $9^{a}$ edição e, desde 1998, quando atraiu 197.221 candidatos vem crescendo até o ano passado quando se inscreveram 3.742.827 candidatos.

É possível que uma das principais razões para o grande aumento nos últimos dois anos seja a criação do Programa Universidade para Todos (PROUNI) que oferece bolsas de estudos para a educação superior. Os participantes do ENEM podem candidatar-se ao PROUNI se satisfizerem os seguintes critérios: apresentar renda familiar per capita até 3 salários mínimos, ter cursado o Ensino Médio em escolas públicas ou em particulares com bolsa integral, ser portador de necessidades especiais e ser professor da rede pública de Educação Básica em efetivo exercício, buscando vagas nos cursos de Licenciatura e Pedagogia. $O$ Ministério da Educação exige também uma nota mínima no ENEM. Por esses critérios estima-se que os alunos dos CPVCs têm no ENEM sua melhor chance de obter vagas na universidade - via PROUNI, desde que, além de satisfazerem aos critérios socioeconômicos 
consigam obter um bom desempenho nas provas. É importante acrescentar que, desde a $1^{a}$ edição do ENEM quando apenas 10 faculdades utilizavam seus resultados para acesso ao ensino superior, em 2006 esse número de faculdades chega a cerca de 500 .

ENEM é um exame elaborado a partir de uma matriz de competências que contempla os Parâmetros Curriculares do Ensino Médio e considera ainda as expectativas de professores universitários e especialistas em seleção e recrutamento de mão de obra, em relação ao perfil de saída dos alunos da Educação Básica no país.

Elaborada de forma interdisciplinar e contextualizada, face aos temas e problemas do dia-adia dos participantes, a prova é composta de 63 itens, cada um deles apresentando uma situação problema inédita. Esta prova avalia cinco competências: domínio de linguagens, compreensão de fenômenos, enfrentamento de problemas, construção de argumentação e elaboração de propostas de intervenção na realidade, que são operacionalizadas em 21 habilidades. A proposta de Redação tem sido elaborada de forma a possibilitar que o participante com base nos subsídios oferecidos pela leitura da coletânea de textos-estímulo organize dados do seu repertório e produza um texto argumentativo-dissertativo. Além das provas, os candidatos ao ENEM respondem a um questionário socioeconômico extenso, composto por 223 itens. As respostas dessas questões permitem traçar um perfil dos candidatos além de estabelecer correlações entre algumas variáveis e as notas obtidas.

A tabela 2, a seguir, apresenta a distribuição dos alunos que participaram do ENEM, por unidades da federação e Distrito Federal, com as respectivas médias de desempenho na prova objetiva.
Tabela 2 - Número de participantes no ENEM 2006 e médias - UF e Distrito Federal.

\begin{tabular}{|c|c|c|}
\hline Brasil/Estados & $\mathrm{N}^{\circ}$ Alunos & Média \\
\hline $\mathrm{BR}$ & 2.783 .968 & 36,9 \\
\hline RS & 146.197 & 39,7 \\
\hline MG & 332.666 & 39,1 \\
\hline SP & 787.499 & 38,9 \\
\hline ES & 63.339 & 38,6 \\
\hline$R$ & 187.623 & 38,6 \\
\hline PR & 148.914 & 38,1 \\
\hline SC & 39.975 & 37,8 \\
\hline DF & 44.082 & 37,7 \\
\hline PE & 116.213 & 36,0 \\
\hline $\mathrm{GO}$ & 70.445 & 35,6 \\
\hline CE & 83.625 & 34,8 \\
\hline MS & 44.512 & 34,8 \\
\hline RN & 48.459 & 34,2 \\
\hline MT & 50.601 & 33,9 \\
\hline PB & 28.939 & 33,6 \\
\hline SE & 19.014 & 33,5 \\
\hline BA & 286.075 & 33,3 \\
\hline PA & 77.304 & 33,2 \\
\hline $\mathrm{PI}$ & 37.950 & 33,1 \\
\hline $\mathrm{RO}$ & 29.164 & 32,7 \\
\hline $\mathrm{AL}$ & 15.960 & 32,3 \\
\hline$R R$ & 9.584 & 31,4 \\
\hline$A P$ & 11.320 & 31,4 \\
\hline$M A$ & 47.163 & 31,3 \\
\hline$A C$ & 5.802 & 31,0 \\
\hline AM & 33.651 & 30,9 \\
\hline $\mathrm{TO}$ & 17.892 & 30,7 \\
\hline
\end{tabular}

$O$ percentual geral de presenças em 2006 foi de 74,38\%. (FUNDAÇÃO CESGRANRIO, 2006). 
Como se pode analisar as médias obtidas pelos candidatos são bastante baixas. A média no Brasil foi de 36,9 em uma escala de 0 a 100. O desempenho médio dos alunos nas diferentes unidades da federação varia em cerca de 9 pontos, sendo o maior desempenho médio exibido pelos candidatos do Rio Grande do Sul e o menor, por Tocantins.

A média dos alunos dos CPVCs na prova objetiva do ENEM foi de 39,9, dois décimos acima da média dos participantes do Rio Grande do Sul. É importante lembrar que a média geral do ENEM abrange também os alunos das escolas particulares e federais - que em todas as avaliações rea- lizadas apresentam, em média, os melhores desempenhos.

Na discussão dos resultados a seguir, as médias, os percentuais de acertos nas questões por habilidades e algumas características socioeconômicas e de escolaridade dos pré-vestibulandos comunitários serão apresentadas comparativamente aos resultados obtidos para o Rio de Janeiro e o Brasil.

A análise do desempenho dos alunos nas questões do ENEM está apresentada no Quadro 2, ordenada decrescentemente pela diferença dos percentuais de acerto entre os alunos dos CPVCs e de todos os participantes no Brasil.

\begin{tabular}{|c|c|c|c|c|c|c|}
\hline Item & Habilidade & CPVCs & $R J$ & BR & CPVCs - RJ & CPVCs - BR \\
\hline 47 & 12 & 0,72 & 0,66 & 0,62 & 0,06 & 0,10 \\
\hline 16 & 18 & 0,49 & 0,43 & 0,39 & 0,06 & 0,10 \\
\hline 22 & 20 & 0,37 & 0,30 & 0,27 & 0,07 & 0,10 \\
\hline 21 & 20 & 0,71 & 0,66 & 0,61 & 0,05 & 0,10 \\
\hline 15 & 21 & 0,69 & 0,62 & 0,59 & 0,07 & 0,10 \\
\hline 17 & 21 & 0,73 & 0,67 & 0,63 & 0,06 & 0,10 \\
\hline 13 & 19 & 0,52 & 0,45 & 0,43 & 0,07 & 0,09 \\
\hline 11 & 19 & 0,55 & 0,49 & 0,46 & 0,06 & 0,09 \\
\hline 18 & 19 & 0,57 & 0,51 & 0,48 & 0,06 & 0,09 \\
\hline 1 & 5 & 0,56 & 0,50 & 0,48 & 0,06 & 0,08 \\
\hline 38 & 8 & 0,60 & 0,55 & 0,52 & 0,05 & 0,08 \\
\hline 55 & 1 & 0,32 & 0,33 & 0,25 & $-0,01$ & 0,07 \\
\hline 44 & 13 & 0,60 & 0,56 & 0,53 & 0,04 & 0,07 \\
\hline 6 & 6 & 0,43 & 0,40 & 0,37 & 0,03 & 0,06 \\
\hline 50 & 8 & 0,38 & 0,36 & 0,32 & 0,02 & 0,06 \\
\hline 7 & 5 & 0,57 & 0,56 & 0,51 & 0,01 & 0,06 \\
\hline 8 & 6 & 0,40 & 0,37 & 0,35 & 0,03 & 0,05 \\
\hline 58 & 7 & 0,34 & 0,32 & 0,29 & 0,02 & 0,05 \\
\hline
\end{tabular}

(Continua) 
(Continuação)

\begin{tabular}{|c|c|c|c|c|c|c|}
\hline 9 & 10 & 0,55 & 0,52 & 0,50 & 0,03 & 0,05 \\
\hline 36 & 11 & 0,39 & 0,35 & 0,34 & 0,04 & 0,05 \\
\hline 14 & 18 & 0,42 & 0,38 & 0,37 & 0,04 & 0,05 \\
\hline 41 & 16 & 0,72 & 0,65 & 0,67 & 0,07 & 0,05 \\
\hline 25 & 2 & 0,54 & 0,53 & 0,50 & 0,01 & 0,04 \\
\hline 39 & 8 & 0,34 & 0,31 & 0,30 & 0,03 & 0,04 \\
\hline 30 & 9 & 0,66 & 0,63 & 0,62 & 0,03 & 0,04 \\
\hline 12 & 10 & 0,62 & 0,59 & 0,58 & 0,03 & 0,04 \\
\hline 5 & 6 & 0,49 & 0,47 & 0,45 & 0,02 & 0,04 \\
\hline 31 & 9 & 0,31 & 0,29 & 0,27 & 0,02 & 0,04 \\
\hline 42 & 12 & 0,41 & 0,38 & 0,37 & 0,03 & 0,04 \\
\hline 20 & 15 & 0,32 & 0,31 & 0,28 & 0,01 & 0,04 \\
\hline 35 & 11 & 0,32 & 0,30 & 0,29 & 0,02 & 0,03 \\
\hline 4 & 18 & 0,49 & 0,44 & 0,46 & 0,05 & 0,03 \\
\hline 34 & 11 & 0,49 & 0,47 & 0,47 & 0,02 & 0,02 \\
\hline 33 & 16 & 0,49 & 0,48 & 0,47 & 0,01 & 0,02 \\
\hline 61 & 1 & 0,35 & 0,34 & 0,33 & 0,01 & 0,02 \\
\hline 2 & 4 & 0,42 & 0,42 & 0,41 & 0,00 & 0,01 \\
\hline 62 & 14 & 0,19 & 0,20 & 0,18 & $-0,01$ & 0,01 \\
\hline 32 & 16 & 0,45 & 0,43 & 0,44 & 0,02 & 0,01 \\
\hline 49 & 17 & 0,41 & 0,43 & 0,40 & $-0,02$ & 0,01 \\
\hline 48 & 21 & 0,35 & 0,35 & 0,34 & 0,00 & 0,01 \\
\hline 63 & 2 & 0,34 & 0,35 & 0,34 & $-0,01$ & 0,00 \\
\hline 57 & 3 & 0,40 & 0,42 & 0,40 & $-0,02$ & 0,00 \\
\hline 45 & 4 & 0,29 & 0,31 & 0,29 & $-0,02$ & 0,00 \\
\hline 46 & 12 & 0,24 & 0,26 & 0,24 & $-0,02$ & 0,00 \\
\hline 37 & 13 & 0,29 & 0,30 & 0,29 & $-0,01$ & 0,00 \\
\hline 59 & 14 & 0,20 & 0,21 & 0,20 & $-0,01$ & 0,00 \\
\hline 52 & 17 & 0,34 & 0,34 & 0,34 & 0,00 & 0,00 \\
\hline 24 & 3 & 0,21 & 0,22 & 0,22 & $-0,01$ & $-0,01$ \\
\hline 27 & 4 & 0,24 & 0,25 & 0,25 & $-0,01$ & $-0,01$ \\
\hline 43 & 13 & 0,32 & 0,32 & 0,33 & 0,00 & $-0,01$ \\
\hline 29 & 20 & 0,26 & 0,28 & 0,27 & $-0,02$ & $-0,01$ \\
\hline
\end{tabular}

(Continua) 
(Continuação)

\begin{tabular}{|c|c|c|c|c|c|c|}
\hline 26 & 15 & 0,40 & 0,44 & 0,42 & $-0,04$ & $-0,02$ \\
\hline 56 & 7 & 0,16 & 0,17 & 0,18 & $-0,01$ & $-0,02$ \\
\hline 10 & 10 & 0,13 & 0,16 & 0,15 & $-0,03$ & $-0,02$ \\
\hline 28 & 1 & 0,18 & 0,20 & 0,20 & $-0,02$ & $-0,02$ \\
\hline 23 & 2 & 0,15 & 0,17 & 0,17 & $-0,02$ & $-0,02$ \\
\hline 51 & 7 & 0,18 & 0,20 & 0,20 & $-0,02$ & $-0,02$ \\
\hline 19 & 15 & 0,12 & 0,16 & 0,14 & $-0,04$ & $-0,02$ \\
\hline 54 & 17 & 0,12 & 0,15 & 0,14 & $-0,03$ & $-0,02$ \\
\hline 3 & 5 & 0,32 & 0,36 & 0,35 & $-0,04$ & $-0,03$ \\
\hline 53 & 3 & 0,21 & 0,25 & 0,24 & $-0,04$ & $-0,03$ \\
\hline 60 & 14 & 0,25 & 0,30 & 0,29 & $-0,05$ & $-0,04$ \\
\hline 40 & 9 & 0,42 & 0,46 & 0,46 & $-0,04$ & $-0,04$ \\
\hline Media & & 0,40 & 0,39 & 0,37 & 0,01 & 0,03 \\
\hline
\end{tabular}

Quadro 2 - Percentual de acertos ordenados nos itens da prova/habilidades - alunos dos CPVCs, RJ, e Brasil e diferenças percentuais - ENEM 2006.

O desempenho dos alunos dos CPVCs é superior aos alunos brasileiros em 40 das 63 questões aplicadas. Os maiores percentuais de acertos estão em 9 questões cujas diferenças variam entre 9 e 10 pontos percentuais.

Em 13 questões as diferenças percentuais situam-se entre 8 e 5 pontos. É interessante analisar que, conforme já apontado nos resultados obtidos no simulado, as questões cujos desempenhos dos alunos dos CPVCs são piores que o Brasil e o Rio de Janeiro são, por exemplo, as questões $n^{\circ}$ $40,60,53,3,54,19,51,23$ cujas habilidades são respectivamente $9,14,3,5,17$, 15, 7 e 2. Tais habilidades relacionam-se basicamente com as áreas de conhecimento da biologia, estatística química, física e geometria. É possível que nessas áreas de conhecimento seja necessário um maior tempo para romper com a falta de pré-requisitos oriundos de trajetórias escolares interrompidas ou de freqüência de escolas públicas, nas quais a falta de professores nessas áreas é uma variável importante. É interessante assinalar também que, no curso de Capacitação oferecido em 2006, o percentual de professores dessas áreas era bem menor do que das áreas de humanas e Língua Portuguesa. Por exemplo, 80 professores de Língua Portuguesa, 90 de Geografia e História participaram do treinamento contra um total de 90 professores das áreas de Biologia, Física, Matemática

A tabela 3 apresenta as médias obtidas pelos alunos dos CPVCs. 
Tabela 3 - Médias dos alunos dos CPVCs - Brasil e Rio de Janeiro do ENEM 2006.

\begin{tabular}{|l|c|c|c|}
\hline & Brasil & $\begin{array}{c}\text { Rio de } \\
\text { Janeiro }\end{array}$ & $\begin{array}{c}\text { Alunos } \\
\text { dos CPVCs }\end{array}$ \\
\hline Prova Objetiva & 36,90 & 38,60 & 39,86 \\
\hline Redação & 52,08 & 53,32 & 58,85 \\
\hline
\end{tabular}

Como se pode notar o desempenho médio dos alunos dos CPVCs é superior ao desempenho médio dos participantes do Rio de Janeiro e do Brasil na prova objetiva e na redação. $\mathrm{Na}$ redação a diferença de desempenho médio dos alunos dos CPVCs para todos os participantes do Brasil é de cerca de 7 pontos.

A seguir serão discutidos os resultados dos alunos dos CPVCs por variáveis de interesse : idade, sexo, cor e renda familiar (FUNDAÇÃO CESGRANRIO, 2006).

A tabela 4 apresenta as médias de desempenho por idade, em anos completos em 31 de julho. A idade é uma variável de interesse no desempenho, pois diferentes estudos têm mostrado uma associação negativa entre atraso e desempenho.

Como se pode ver as médias dos alunos dos CPVCs se mantêm praticamente constantes até a idade de 21 anos (cerca de 41,00 ) enquanto as médias do Brasil e do Rio de Janeiro caem com a progressão da idade (de 40,49 a 35,74 e de 45,51 a 36,06 , respectivamente)

Tabela 4 - Idade dos alunos dos CPVCs - Brasil e Rio de Janeiro do ENEM 2006

\begin{tabular}{|l|r|r|l|r|r|r|r|r|r|}
\hline & \multicolumn{3}{|c|}{ BRASIL } & \multicolumn{3}{|c|}{ RIO DE JANEIRO } & \multicolumn{3}{c|}{ CPVCs } \\
\hline Idade & $\mathrm{N}^{\circ}$ & $\%$ & Média & $\mathrm{N}^{\circ}$ & $\%$ & Média & $\mathrm{N}^{\circ}$ & $\%$ & Média \\
\hline 16 & 228008 & 8,19 & 40,49 & 12968 & 6,91 & 45,51 & 106 & 4,52 & 40,55 \\
\hline 17 & 618682 & 22,22 & 38,93 & 44244 & 23,58 & 42,99 & 377 & 16,06 & 41,14 \\
\hline 18 & 373286 & 13,41 & 37,11 & 30543 & 16,28 & 38,99 & 406 & 17,3 & 42,3 \\
\hline 19 & 260718 & 9,36 & 36,16 & 17520 & 9,34 & 36,94 & 220 & 9,37 & 41,16 \\
\hline 20 & 199116 & 7,15 & 35,86 & 12037 & 6,42 & 36,27 & 191 & 8,14 & 40,26 \\
\hline 21 & 149906 & 5,38 & 35,74 & 8341 & 4,45 & 36,06 & 136 & 5,79 & 40,95 \\
\hline 22 & 122802 & 4,41 & 35,88 & 6710 & 3,58 & 36,19 & 96 & 4,09 & 39,91 \\
\hline 23 & 106866 & 3,84 & 35,94 & 5522 & 2,94 & 36 & 81 & 3,45 & 38,11 \\
\hline 24 & 92355 & 3,32 & 35,81 & 5037 & 2,68 & 35,94 & 81 & 3,45 & 37,7 \\
\hline $24-29$ & 274952 & 9,88 & 35,41 & 15836 & 8,44 & 35,27 & 242 & 10,31 & 37,96 \\
\hline$>29$ & 318720 & 11,45 & 34,01 & 26677 & 14,22 & 33,66 & 400 & 17,04 & 36,65 \\
\hline SI & 38557 & 1,38 & 39,03 & 2188 & 1,17 & 41,24 & 11 & 0,47 & 38,38 \\
\hline
\end{tabular}

A tabela 5 referente ao sexo revela que a média na prova objetiva dos alunos do sexo masculino é maior do que as do sexo feminino em todos os casos estudados. Chama-se a atenção que o número de alunos do sexo feminino que fizeram o ENEM é muito maior do que os alunos do sexo masculino. É possível que essa predominância tenha um efeito no desempenho. 
Tabela 5 - Sexo dos alunos dos CPVCs - Brasil e Rio de Janeiro do ENEM 2006.

\begin{tabular}{|l|r|r|r|r|r|r|r|r|r|}
\hline & \multicolumn{3}{|c|}{ BRASIL } & \multicolumn{3}{c|}{ RIO DE JANEIRO } & \multicolumn{3}{c|}{ CPVCs } \\
\hline & $N^{\circ}$ & $\%$ & Média & $N^{\circ}$ & $\%$ & Média & $N^{\circ}$ & $\%$ & Média \\
\hline Masculino & 1051503 & 37,77 & 39,83 & 70646 & 37,65 & 41,59 & 710 & 30,25 & 43,05 \\
\hline Feminino & 1731949 & 62,21 & 35,12 & 116622 & 62,16 & 36,77 & 1634 & 69,62 & 38,47 \\
\hline S/Inf. & 516 & 0,02 & 32,58 & 355 & 0,19 & 33,09 & 3 & 0,13 & 37,04 \\
\hline
\end{tabular}

As análises a seguir utilizam o questionário socioeconômico do ENEM e os percentuais apresentados são em relação ao total de participantes. Ressalta-se que cerca de $15 \%$ dos participantes do ENEM no Brasil não responderam ao questionário. No Estado do Rio de Janeiro, esse percentual sobe para $25 \%$ e entre os alunos dos CPVCs é de $12 \%$.

As Tabelas 6 e 7 apresentarão os perfis e os resultados dos desempenhos médios dos alunos segundo a sua declaração de cor e de renda familiar.

Na tabela 6, observa-se a diferença do perfil dos alunos dos CPVCs em relação aos participantes no Brasil e no Estado do Rio de Janeiro. Enquanto 37\% dos participantes do ENEM no Brasil se consideram brancos e $40 \%$ no Estado do Rio de Janeiro, esse percentual cai para somente $24 \%$ entre os alunos dos CPVCs. Por outro lado $21 \%$ dos alunos dos
CPVCs se consideram de cor preta versus somente $6 \%$ dos participantes no Rio de Janeiro. No Brasil, esse percentual é de $11 \%$. A média dos alunos dos CPVCs que se consideram pretos é menor que a média dos alunos dos CPVCs que se consideram pardos ou brancos, mas essa média de 38,08 é maior que a média dos participantes brasileiros que se consideram brancos, 37,3.

Na tabela 7, observa-se que enquanto quase $52 \%$ dos alunos dos CPVCs têm renda familiar menor ou igual a 2 salários mínimos, esse percentual cai para quase $40 \%$ no Estado do Rio de Janeiro e para $46 \%$ no Brasil. Surpreendentemente, as médias na prova objetiva por faixa de renda, dos participantes do ENEM no Brasil e no Estado do Rio de Janeiro, apresentam pouca variação, apesar de uma ligeira tendência de crescimento. Já entre os alunos dos CPVCs, observa-se uma acentuada tendência de crescimento com a faixa de renda.

Tabela 6 - Cor dos alunos dos CPVCs - Brasil e Rio de Janeiro, do ENEM 2006.

\begin{tabular}{|l|r|r|r|r|r|r|r|r|r|}
\hline & \multicolumn{3}{|c|}{ BRASIL } & \multicolumn{3}{|c|}{ RIO DE JANEIRO } & \multicolumn{3}{|c|}{ CPVCs } \\
\hline Cor & $N^{\circ}$ & $\%$ & Média & $N^{\circ}$ & $\%$ & Média & $N^{\circ}$ & $\%$ & Média \\
\hline BRA & 1027013 & 36,89 & 37,3 & 75807 & 40,4 & 38,58 & 571 & 24,33 & 41,95 \\
PAR & 951350 & 34,17 & 36,52 & 47111 & 25,11 & 37,87 & 932 & 39,71 & 39,43 \\
PRE & 299574 & 10,76 & 36,09 & 10811 & 5,76 & 38,02 & 502 & 21,39 & 38,08 \\
AMA & 82944 & 2,98 & 36,52 & 4602 & 2,45 & 37,96 & 57 & 2,43 & 36,76 \\
IND & 23506 & 0,84 & 36,44 & 1433 & 0,76 & 36,34 & 19 & 0,81 & 37,09 \\
Em branco & 397226 & 14,27 & 37,5 & 47744 & 25,45 & 39,51 & 265 & 11,29 & 41,13 \\
S/Inf. & 2355 & 0,08 & 37,16 & 115 & 0,06 & 38,72 & 1 & 0,04 & 20,63 \\
\hline
\end{tabular}


Tabela 7 - Renda Familiar (em salários mínimos) dos alunos dos CPVCs - Brasil e Rio de Janeiro do ENEM 2006.

\begin{tabular}{|l|r|r|r|r|r|r|r|r|r|}
\hline & \multicolumn{4}{|c|}{ BRASIL } & \multicolumn{3}{c|}{ RIO DE JANEIRO } & \multicolumn{3}{|c|}{ CPVCs } \\
\hline Renda & $N^{\circ}$ & $\%$ & Média & $N^{\circ}$ & $\%$ & Média & $N^{\circ}$ & $\%$ & Média \\
\hline$<1$ & 382977 & 13,76 & 36,45 & 20036 & 10,68 & 37,66 & 292 & 12,44 & 36,43 \\
\hline $1-2$ & 894812 & 32,14 & 36,71 & 54203 & 28,89 & 38,17 & 918 & 39,11 & 38,65 \\
\hline $2-5$ & 744324 & 26,74 & 36,95 & 44983 & 23,98 & 38,56 & 732 & 31,19 & 41,56 \\
\hline $5-10$ & 208399 & 7,49 & 37,17 & 12849 & 6,85 & 38,87 & 109 & 4,64 & 44,44 \\
\hline $10-30$ & 91237 & 3,28 & 37,26 & 4885 & 2,6 & 37,9 & 8 & 0,34 & 52,78 \\
\hline $30-50$ & 13832 & 0,5 & 37,12 & 554 & 0,3 & 37,34 & 0 & 0 & NA \\
\hline$>50$ & 7825 & 0,28 & 37,25 & 312 & 0,17 & 37,74 & 1 & 0,04 & 71,43 \\
\hline Nenhuma & 37854 & 1,36 & 36,38 & 1762 & 0,94 & 36,76 & 13 & 0,55 & 36,26 \\
\hline Em branco & 399876 & 14,36 & 37,5 & 47889 & 25,52 & 39,5 & 273 & 11,63 & 40,92 \\
\hline Inválido & 2832 & 0,1 & 37,03 & 150 & 0,08 & 39,79 & 1 & 0,04 & 26,98 \\
\hline
\end{tabular}

Outras variáveis estudadas, correlacionadas com o desempenho dos alunos referem-se às questões de número 72 a
78 do questionário socioeconômico que tratam da trajetória escolar dos alunos e tipo de escola por eles freqüentada

Tabela 8 - Tempo de conclusão do Ensino Fundamental dos alunos dos CPVCs - Brasil e Rio de Janeiro do ENEM 2006.

\begin{tabular}{|l|r|r|r|r|r|r|r|r|r|}
\hline & \multicolumn{4}{|c|}{ BRASIL } & \multicolumn{3}{|c|}{ RIO DE JANEIRO } & \multicolumn{3}{|c|}{ CPVCs } \\
\hline Anos & $N^{\circ}$ & $\%$ & Média & $N^{\circ}$ & $\%$ & Média & $N^{\circ}$ & $\%$ & Média \\
\hline$<8$ anos & 394425 & 14,17 & 34,33 & 32296 & 17,21 & 35,93 & 398 & 16,96 & 37,27 \\
\hline 8 anos & 1327041 & 47,67 & 39,87 & 84223 & 44,89 & 42,33 & 1094 & 46,61 & 41,36 \\
\hline 9 anos & 357003 & 12,82 & 33,89 & 27339 & 14,57 & 36,04 & 339 & 14,44 & 40,16 \\
\hline 10 anos & 135185 & 4,86 & 32,68 & 10617 & 5,66 & 33,9 & 123 & 5,24 & 36,02 \\
\hline 11 anos & 66417 & 2,39 & 31,47 & 4503 & 2,4 & 32,74 & 60 & 2,56 & 36,48 \\
\hline$>11$ anos & 91112 & 3,27 & 29,83 & 7560 & 4,03 & 30,79 & 54 & 2,3 & 34,42 \\
\hline Em branco & 406798 & 14,61 & 36,29 & 20791 & 11,08 & 37,37 & 276 & 11,76 & 40,65 \\
\hline Inválido & 5987 & 0,22 & 32,11 & 294 & 0,16 & 32,83 & 3 & 0,13 & 46,56 \\
\hline
\end{tabular}

número de anos necessários para a conclusão do Ensino Fundamental e também do Médio (tabela 10) é indicativo de repetência e/ou interrupção do processo escolar. $\bigcirc$ desempenho dos alunos é afetado por essas variáveis, ou seja, quanto maior o número de anos para concluir um ciclo de estudos, pior o desempenho (KLEIN, 2006). Como se pode ver na idade correta (8 anos) a média no ENEM é maior em todos os três grupos estudados. Nos alunos dos 
CPVCs o tempo de conclusão de 9 anos (provavelmente uma repetência) não apresenta efeito significativo na média enquanto nos dois outros grupos - Brasil/ Rio de Janeiro, observa-se uma queda de cerca de 6 pontos na média dos alunos que concluíram em 9 anos, em relação aos que concluíram em 8 anos.

Os alunos que cursaram o Ensino Fundamental em escola particular têm, em média, melhor desempenho. Nos alunos do CPVCs a média é também maior, entretanto as diferenças entre as médias dos que cursaram somente a escola pú- blica e os que cursaram somente a particular é apenas de 5 pontos percentuais, enquanto, no Brasil, esta diferença é de cerca de 14 pontos.

No Estado do Rio de Janeiro, a presença da escola particular é muito maior que a média brasileira. Observa-se que $18 \%$ dos participantes do Estado do Rio de Janeiro estudaram somente em escola particular, percentual que cai para 9\% (metade) no Brasil. Mesmo assim, somente $10 \%$ dos alunos dos CPVCs fizeram o EF somente em escola particular.

Tabela 9 - Tipo de escola em que cursaram o Ensino Fundamental os alunos dos CPVCs - Brasil e Rio de Janeiro do ENEM 2006

\begin{tabular}{|l|r|r|r|r|r|r|r|r|r|}
\hline & \multicolumn{3}{|c|}{ BRASIL } & \multicolumn{3}{c|}{ RIO DE JANEIRO } & \multicolumn{3}{c|}{ CPVCs } \\
\hline Tipo de Escola & $\mathrm{N}^{\circ}$ & $\%$ & Média & $\mathrm{N}^{\circ}$ & $\%$ & Média & $\mathrm{N}^{\circ}$ & $\%$ & Média \\
\hline $\begin{array}{l}\text { Somente } \\
\text { pública }\end{array}$ & 1877282 & 67,43 & 34,76 & 104758 & 55,83 & 34,93 & 1423 & 60,63 & 38,58 \\
\hline $\begin{array}{l}\text { Parte em } \\
\text { pública }\end{array}$ & 249015 & 8,94 & 40,31 & 27405 & 14,61 & 39,44 & 388 & 16,53 & 41,51 \\
\hline $\begin{array}{l}\text { Somente em } \\
\text { particular }\end{array}$ & 246106 & 8,84 & 50,69 & 34252 & 18,26 & 49,69 & 245 & 10,44 & 43,83 \\
\hline $\begin{array}{l}\text { Somente em } \\
\text { indígena }\end{array}$ & 1932 & 0,07 & 34,24 & 160 & 0,09 & 40,79 & 2 & 0,09 & 37,3 \\
\hline $\begin{array}{l}\text { Parte em } \\
\text { indígena }\end{array}$ & 1448 & 0,05 & 30,63 & 69 & 0,04 & 38,56 & 0 & 0 & $N A$ \\
\hline Em branco & 406779 & 14,61 & 36,39 & 20887 & 11,13 & 37,48 & 288 & 12,27 & 40,59 \\
\hline Inválido & 1406 & 0,05 & 34,73 & 92 & 0,05 & 34,92 & 1 & 0,04 & 30,16 \\
\hline
\end{tabular}

A tabela 10 mostra a relação entre as médias e o ano de conclusão do Ensino Médio.

Observa-se que o melhor desempenho médio nos 3 grupos considerados, Brasil, Estado do Rio de Janeiro e alunos dos CPVCs, está entre os participantes que concluíram em 2005, provavelmente indicando que passaram o ano após a conclusão do Ensino Médio preparando-se. Chama a atenção que os participantes que responderam às categorias após 2006 e no $1^{\circ}$ semestre de 2006 apresentam um desempenho médio bem inferior. $\bigcirc 1^{\circ}$ caso refere-se aos alunos que em 2006 
ainda estavam no $1^{\circ}$ ou $2^{\circ}$ ano do Ensino Médio e no $2^{\circ}$ caso, provavelmente, refere-se aos participantes que acabaram - Ensino Médio e, no meio do ano, pela
Educação de Jovens e Adultos (EJA). É surpreendente que o desempenho médio dos que concluíram em 2004, ou antes, é praticamente o mesmo.

Tabela 10 - Ano de Conclusão do Ensino Médio dos alunos dos CPVCs

- Brasil e Rio de Janeiro do ENEM 2006.

\begin{tabular}{|l|r|r|r|r|r|r|r|r|r|}
\hline & \multicolumn{3}{|c|}{ BRASIL } & \multicolumn{3}{c|}{ RIO DE JANEIRO } & \multicolumn{3}{|c|}{ CPVCs } \\
\hline Ano & $N^{\circ}$ & $\%$ & Média & $N^{\circ}$ & $\%$ & Média & $N^{\circ}$ & $\%$ & Média \\
\hline Após 2006 & 322321 & 11,58 & 32,76 & 26797 & 14,28 & 34,83 & 128 & 5,45 & 36 \\
\hline $\begin{array}{l}2^{\circ} \text { semestre } \\
\text { de 2006 }\end{array}$ & 808572 & 29,04 & 37,54 & 71114 & 37,9 & 39,87 & 514 & 21,9 & 40,11 \\
\hline $\begin{array}{l}1^{\circ} \text { semestre } \\
\text { de 2006 }\end{array}$ & 46923 & 1,69 & 32,58 & 4163 & 2,22 & 34,36 & 27 & 1,15 & 32,8 \\
\hline 2005 & 285189 & 10,24 & 38,39 & 18518 & 9,87 & 40,99 & 437 & 18,62 & 40,53 \\
\hline 2004 & 200763 & 7,21 & 38,02 & 11693 & 6,23 & 39,1 & 254 & 10,82 & 39,75 \\
\hline 2003 & 146683 & 5,27 & 37,94 & 7800 & 4,16 & 38,96 & 175 & 7,46 & 40,01 \\
\hline 2002 & 107710 & 3,87 & 37,68 & 4867 & 2,59 & 38,9 & 94 & 4,01 & 39,61 \\
\hline 2001 & 90096 & 3,24 & 37,79 & 4178 & 2,23 & 38,51 & 77 & 3,28 & 39,93 \\
\hline $1999-2000$ & 141285 & 5,07 & 37,56 & 6057 & 3,23 & 38,43 & 111 & 4,73 & 40,1 \\
\hline Antes de 1999 & 213577 & 7,67 & 37,95 & 11254 & 6 & 38,41 & 244 & 10,4 & 39,54 \\
\hline Em branco & 405648 & 14,57 & 36,29 & 20440 & 10,89 & 37,34 & 276 & 11,76 & 40,78 \\
\hline Inválido & 15201 & 0,55 & 36,19 & 742 & 0,4 & 36,74 & 10 & 0,43 & 47,14 \\
\hline
\end{tabular}

Tabela 11 - Tempo de conclusão do Ensino Médio dos alunos dos CPVCs - Brasil e Rio de Janeiro do ENEM 2006

\begin{tabular}{|l|r|r|r|r|r|r|r|r|r|}
\hline & \multicolumn{3}{|c}{ BRASIL } & \multicolumn{3}{c|}{ RIO DE JANEIRO } & \multicolumn{3}{c|}{ CPVCs } \\
\hline Anos & $N^{\circ}$ & $\%$ & Média & $N^{\circ}$ & $\%$ & Média & $N^{\circ}$ & $\%$ & Média \\
\hline$<3$ anos & 203507 & 7,31 & 31,92 & 11531 & 6,15 & 33,19 & 112 & 4,77 & 38,14 \\
3 anos & 1791971 & 64,37 & 38,15 & 120179 & 64,05 & 40,1 & 1574 & 67,06 & 40,12 \\
4 anos & 247354 & 8,88 & 34,56 & 24492 & 13,05 & 36,08 & 265 & 11,29 & 39,6 \\
5 anos & 51209 & 1,84 & 34,61 & 4493 & 2,39 & 35,57 & 68 & 2,9 & 38,05 \\
6 anos & 17348 & 0,62 & 33,43 & 1426 & 0,76 & 34,66 & 13 & 0,55 & 34,68 \\
$>6$ anos & 39703 & 1,43 & 31,92 & 3468 & 1,85 & 33,72 & 26 & 1,11 & 38,03 \\
Em branco & 430557 & 15,47 & 36,29 & 21916 & 11,68 & 37,51 & 285 & 12,14 & 40,3 \\
Inválido & 2319 & 0,08 & 33,35 & 118 & 0,06 & 34,29 & 4 & 0,17 & 29,37 \\
\hline
\end{tabular}


Na análise da tabela 11, chega-se às mesmas conclusões que na análise da tabela 7 - tempo de conclusão do Ensino Fundamental. Os alunos dos 3 grupos estudados que concluíram o Ensino Médio na idade correta (3 anos) têm melhor desempenho. Novamente verifica-se que a repetência não contribui para melhorar os níveis de aprendizagem dos alunos.

O turno em que os alunos cursam o Ensino Médio também se relaciona com o desempenho. As médias dos alunos que fazem o Ensino Médio no noturno são mais baixas do que as daqueles que cursam o diurno, nos 3 grupos estudados. A diferença das médias dos alunos dos CPVCs que cursam o diurno e o noturno exclusivamente é de apenas 5 pontos, enquanto, no Brasil, essa diferença é de 15 pontos.

Sabe-se que, em geral, os alunos que estudam no turno noturno estão atrasados, isto é, têm defasagem idade-série, e têm um nível socioeconômico mais baixo. Estudar nesse turno só faz piorar a situação.

Tabela 12 - Turno em que cursam o Ensino Médio os alunos dos CPVCs - Brasil e Rio de Janeiro do ENEM 2006.

\begin{tabular}{|l|r|r|r|r|r|r|r|r|r|}
\hline & \multicolumn{3}{|c|}{ BRASIL } & \multicolumn{3}{c|}{ RIO DE JANEIRO } & \multicolumn{3}{c|}{ CPVCs } \\
\hline Turno & $\mathrm{N}^{\circ}$ & $\%$ & Média & $\mathrm{N}^{\circ}$ & $\%$ & Média & $\mathrm{N}^{\circ}$ & $\%$ & Média \\
\hline $\begin{array}{l}\text { Somente } \\
\text { diurno }\end{array}$ & 1145314 & 41,14 & 40,01 & 92055 & 49,06 & 41,62 & 1148 & 48,91 & 41,37 \\
\hline $\begin{array}{l}\text { Maior parte } \\
\text { diurno }\end{array}$ & 302414 & 10,86 & 36,9 & 21658 & 11,54 & 39,93 & 285 & 12,14 & 39,82 \\
\hline $\begin{array}{l}\text { Somente } \\
\text { noturno }\end{array}$ & 681572 & 24,48 & 32,93 & 40347 & 21,5 & 32,73 & 479 & 20,41 & 36,44 \\
\hline $\begin{array}{l}\text { Maior parte } \\
\text { noturno }\end{array}$ & 238878 & 8,58 & 34,49 & 12193 & 6,5 & 34,63 & 150 & 6,39 & 37,8 \\
\hline Em branco & 413792 & 14,86 & 36,25 & 21275 & 11,34 & 37,39 & 285 & 12,14 & 40,61 \\
\hline Inválido & 1998 & 0,07 & 31,3 & 95 & 0,05 & 31,9 & 0 & 0 & NA \\
\hline
\end{tabular}

A tabela 13 apresenta as médias dos alunos pelo tipo de escola em que freqüentaram o Ensino Médio. As conclusões são as mesmas da tabela 8 do Ensino Fundamental, tanto em relação ao percentual de alunos que estudam por tipo de escola quanto a diferença de médias entre os que estudaram somente em escola particular e os que estudaram somente em escola pública. 
Tabela 13 - Tipo de escola que cursaram o Ensino Médio os alunos dos CPVCs - Brasil e Rio de Janeiro do ENEM 2006.

\begin{tabular}{|l|r|r|r|r|r|r|r|r|r|}
\hline & \multicolumn{3}{|c|}{ BRASIL } & \multicolumn{3}{c|}{ RIO DE JANEIRO } & \multicolumn{3}{|c|}{ CPVCs } \\
\hline Tipo de Escola & $\mathrm{N}^{\circ}$ & $\%$ & Média & $\mathrm{N}^{\circ}$ & $\%$ & Média & $\mathrm{N}^{\circ}$ & $\%$ & Média \\
\hline $\begin{array}{l}\text { Somente em } \\
\text { pública }\end{array}$ & 1971354 & 70,81 & 34,94 & 122586 & 65,34 & 35,86 & 1693 & 72,13 & 39,56 \\
\hline $\begin{array}{l}\text { Maior parte } \\
\text { em pública }\end{array}$ & 80060 & 2,88 & 36,84 & 6833 & 3,64 & 37,06 & 69 & 2,94 & 36,3 \\
\hline $\begin{array}{l}\text { Somente em } \\
\text { particular }\end{array}$ & 285507 & 10,26 & 50,57 & 33879 & 18,06 & 49,15 & 241 & 10,27 & 42,2 \\
\hline $\begin{array}{l}\text { Maior parte } \\
\text { em particular }\end{array}$ & 40134 & 1,44 & 41,75 & 3857 & 2,06 & 39,91 & 62 & 2,64 & 39,55 \\
\hline $\begin{array}{l}\text { Somente em } \\
\text { indígena }\end{array}$ & 1362 & 0,05 & 33,26 & 112 & 0,06 & 40,09 & 1 & 0,04 & 26,98 \\
\hline $\begin{array}{l}\text { Maior parte } \\
\text { em indígena }\end{array}$ & 1163 & 0,04 & 33,2 & 104 & 0,06 & 37,45 & 1 & 0,04 & 52,38 \\
\hline Em branco & 401676 & 14,43 & 36,37 & 20071 & 10,7 & 37,56 & 278 & 11,84 & 40,63 \\
\hline $\begin{array}{l}\text { Inválido } \\
\text { Snvido }\end{array}$ & 2712 & 0,1 & 35,9 & 181 & 0,1 & 38,97 & 2 & 0,09 & 35,71 \\
\hline
\end{tabular}

O desempenho dos alunos que concluíram o Ensino Médio na modalidade educação de jovens e adultos é bem mais baixo do que os que cursaram o Ensino Regular ou o Ensino Técnico/Profissional. Nos CPVCs, o percentual de alunos que terminaram o Ensino médio pela Educação de Jovens e Adultos é menor que no Brasil e no Rio de Janei- ro, mas surpreendente é o percentual de alunos dos CPVCs que terminaram no ensino técnico/profissional, 19\%, enquanto no Brasil é de somente $8 \%$ e no Estado do Rio de Janeiro de 16\%. A média dos alunos dos CPVCs que cursaram o Ensino Técnico é maior que a média dos alunos dos CPVCs que cursaram o Ensino Regular.

Tabela 14 - Modalidade de ensino de conclusão do Ensino Médio dos alunos dos CPVCs - Brasil e Rio de Janeiro do ENEM 2006.

\begin{tabular}{|l|r|r|r|r|r|r|r|r|r|}
\hline & \multicolumn{3}{|c|}{ BRASIL } & \multicolumn{3}{c|}{ RIO DE JANEIRO } & \multicolumn{3}{c|}{ CPVCs } \\
\hline Modalidade & $\mathrm{N}^{\circ}$ & $\%$ & Média & $\mathrm{N}^{\circ}$ & $\%$ & Média & $\mathrm{N}^{\circ}$ & $\%$ & Média \\
\hline Ensino regular & 1920389 & 68,98 & 37,53 & 121380 & 64,69 & 39,13 & 1467 & 62,51 & 39,17 \\
\hline $\begin{array}{l}\text { Educação } \\
\text { jovens e adultos }\end{array}$ & 222877 & 8,01 & 32,33 & 14986 & 7,99 & 33,77 & 137 & 5,84 & 37,34 \\
\hline $\begin{array}{l}\text { Ensino técnico/ } \\
\text { profisional }\end{array}$ & 213644 & 7,67 & 37,53 & 29182 & 15,55 & 39,66 & 454 & 19,34 & 42,36 \\
\hline Em branco & 425709 & 15,29 & 36,15 & 21976 & 11,71 & 37,34 & 288 & 12,27 & 40,56 \\
\hline Inválido & 1349 & 0,05 & 37,34 & 99 & 0,05 & 40,85 & 1 & 0,04 & 46,03 \\
\hline
\end{tabular}




\section{Conclusões}

Os autores do trabalho acreditam que os resultados obtidos pelos alunos dos CPVCs no ENEM 2006 possam, em certa medida, ser atribuídos ao processo de capacitação dos seus professores e às demais atividades desenvolvidas ao longo do processo. Embora, a rigor, a pesquisa não tenha sido desenhada para medir os ganhos de aprendizagem após a intervenção pedagógica iá que não havia dados disponíveis do desempenho dos alunos dos CPVCs nas edições anteriores do ENEM - há indícios de que os resultados aqui apresentados possam ser creditados ao processo de capacitação aliados a outros fatores. $\bigcirc$ primeiro deles é, sem dúvida, a motivação dos atores do processo - alunos e professores - ambos voluntários, que se esforçam para alcançar seus objetivos. A motivação também se expressa no pedido de continuidade do proje- to em 2007, quando um novo curso de capacitação dos professores está sendo ministrado no período de março a junho de 2007.

$O$ segundo fator que deve ser levado em conta nesses resultados é a vivência, pelos alunos, do processo de empoderamento representado pelo recebimento de um serviço pedagógico nunca antes ocorrido nas suas trajetórias escolares. Muitos alunos chegaram a verbalizar esse sentimento aos seus professores.

Por fim, os autores querem acrescentar que os resultados obtidos pelos alunos dos CPVCs no ENEM 2006 anima-os a prosseguir nesta linha de trabalho e aprofundar a pesquisa, analisando, no próximo ano, o desempenho acadêmico dos alunos dos CPVCs nos diferentes cursos universitários a que tiveram acesso.

\section{Referências}

CARVAlHO, J. C. Cursos Pré-Vestibulares Comunitários e seus condicionantes pedagógicos: um diagnóstico delineado com base em surveys e em práticas institucionais. Cadernos de Pesquisa, São Paulo, n. 128, ago. 2006.

FUNDAÇÃO CESGRANRIO. Relatório técnico do ENEM 2006. Rio de Janeiro, 2006.

KLEIN, R.; FONTANIVE, N.; ELLIOT, L. Exame Nacional do Ensino Médio: tecnologia e principais resultados em 2005. Revista Electrónica Iberoamericana Sobre Calidad Eficácia y Cambio em Educación. v. 5, p. 116-131, 2007. Disponível em:

$<$ http://www.rinace.net/reicepres.htm>. Acesso em: 3 ago. 2007.

KLEIN, R. Como está a educação no Brasil? $\bigcirc$ que fazer?. Ensaio: avaliação e políticas públicas em educação, Rio de Janeiro, v. 14, n. 51, p. 139-172, abr./jun. 2006.

Recebido em: 19/07/2007

Aceito para publicação em: 27/07/2007 